\title{
Die Grenzen der Erkenntnis im Reallabor
}

\author{
Ausgehend von der Einsicht, dass künftigen \\ Generationen durch unser gegenwärtiges \\ Wirtschaften irreversible Nachteile drohen, wurde \\ die Literatur zur Relevanz der wirtschaftlichen \\ Entwicklung für die Verwirklichung umweltpoli- \\ tischer Ziele aufgearbeitet. Welche Erkenntnisse \\ ergeben sich hierfür? \\ Von Christoph M. Schmidt
}

D as Umweltbundesamt hat mit diesem Forschungsauftrag zur Literaturstudie zum einen die in der Politikberatung bisher kaum berücksichtigte Literatur der Diskurse zu Postwachstum und Degrowth zum Gegenstand „auf Augenhöhe“ gemacht. Zum anderen hat es dem Projektteam die Gelegenheit eröffnet, das Zerrbild der „Mainstream“-Volkswirtschaftslehre als einer realitätsfernen Formelwissenschaft in überzeugender Weise zu korrigieren.

Von besonderer Bedeutung für das praktische politische Handeln sind dabei die aus dem Projekt erwachsenden Schlussfolgerungen darüber, welche konkreten Eingriffe dazu geeignet sind, das weithin unbestrittene Ziel der Einhaltung planetarer Grenzen zu erreichen. Sie beruhen auf einer kritischen kontrastierenden Gegenüberstellung unterschiedlicher Denkansätze und darauf bezogener empirischer Befunde und reflektieren die Verständigungsleistung eines Autor/innenteams, das sich dem Thema aus recht unterschiedlichen Perspektiven genähert hat. Dabei wurden zunächst die zwei innerhalb dieses Diskurses besonders prominenten - und antagonistischen - Positionen, Green Growth und Degrowth, aufgrund der mangelnden empirischen Basis für ihre jeweiligen apodiktischen Prognosen und daraus abgeleiteten Schlussfolgerungen entzaubert und davon ausgehend mit der „vorsorgeorientierten Postwachstumsposition“ eine eigenständige pragmatische Position formuliert.

Auf Basis dieser „vorsorgeorientierten Postwachstumsposition" werden in Petschow et al. (2018) drei zentrale Forderungen an die Politik abgeleitet. Es ist äußerst bemerkenswert, dass die erste Forderung, die Internalisierung externer Effekte vor allem mithilfe marktbasierter Instrumente (wie cap \& trade-Systeme oder einer Besteuerung von Emissionen) anzustreben, als inhaltliche Schnittmenge identifiziert wurde. Denn die im Projektverlauf analysierten Positionen des Spektrums von Green Growth bis Degrowth weisen erhebliche Unterschiede im Bereich ihrer charakteristischen Annahmen auf. Damit er- hält diese erste Forderung erhebliches Gewicht und stützt nicht zuletzt interdisziplinäre Schlussfolgerungen des Diskurses zur Energiewende (Edenhofer et al. 2018; Appelrath et al. 2015).

Bei der Umsetzung dieser Forderung wird es aber entscheidend darauf ankommen, dass die Maßnahmen möglichst im internationalen oder gar globalen Kontext, zumindest aber in einer hinreichend großen Allianz europäischer Staaten vorangetrieben werden. Nationale Alleingänge bergen das in der Klimapolitik unter dem Stichwort carbon leakage diskutierte Risiko, dass Eingriffe, die eigentlich zur Internalisierung externer Effekte dienen sollen, in einer globalisierten Wirtschaft lediglich zu einer Verlagerung der entsprechenden Aktivitäten und der damit verbundenen Emissionen ins Ausland führen. Ein umweltpolitischer Vorteil wäre damit nicht verbunden, wohl aber ein Wohlfahrtsverlust durch Einbußen bei Beschäftigung und Wertschöpfung.

Aus ökonomischer Sicht fällt es ebenfalls leicht, sich der dritten Forderung ohne Vorbehalte anzuschließen. Denn das Plädoyer für die Suche nach Potenzialen zur Reduzierung der Wachstumsabhängigkeit insbesondere der Sozialversicherungssysteme bedeutet letztlich, dass deren gesellschaftlich gewünschtes Leistungsniveau mit einem möglichst geringen Einsatz knapper (materieller) Ressourcen erreicht werden soll. In der Konsequenz entspricht diese Forderung dem geradezu ur-ökonomischen Postulat der Effizienz. So forscht das RWI seit vielen Jahren in anwendungsorientierten Projekten gemeinsam mit Praxispartnern etwa im Gesundheitswesen zu Fragen, wie Effizienzreserven durch eine bessere Verzahnung von ambulantem und stationärem Sektor mobilisiert werden können, oder wie sich durch das Zusammenwirken von professioneller Pflege durch Fachkräfte und informeller Pflege durch Angehörige verschiedene Ergebnisgrößen wirksam verbessern lassen. Wenngleich diese Projekte am RWI nicht unter der Überschrift „Reduzierung der Wachstumsabhängigkeit" geführt werden, so dürften sie in ihrer inhaltlichen Substanz doch dem sehr nahekommen, was das Projektteam mit der dritten Forderung der vorsorgeorientierten Postwachstumsposition intendiert.

\section{Möglichkeiten der Erkenntnis im Reallabor ...}

Es gibt allerdings gute Gründe, der zweiten Forderung, neue Pfade der gesellschaftlichen Entwicklung in sogenannten Reallaboren auszuloten, mit einer gewissen Skepsis zu begegnen. Bei aller Euphorie über die Potenziale von Reallaboren, ist es aus meiner Sicht ebenso wichtig, ihre Grenzen zu beachten. Es kann offensichtlich nicht darum gehen, einer vermeint- 
lichen Avantgarde einen zeitlich unbefristeten Experimentierraum zur Verfügung zu stellen - quasi als subventionierten Nährboden für die erhoffte Emergenz eines nachhaltigen Lebensstils, der sich dann trotz der geschützten und mithin artifiziellen Treibhausbedingungen seiner Entstehung flächendeckend ausbreiten können soll. In Ermangelung einer klaren Konkretisierung innerhalb der Studie sollten Reallabore daher in Anlehnung an das Bundeswirtschaftsministerium vielmehr als zeitlich und räumlich begrenzte Experimentierräume verstanden werden, in denen (soziale) Innovationen und Regulierungselemente unter realen Bedingungen erprobt werden können (Hielscher et al. 2018).

Reallabore können vor allem dann ein vielversprechender Ansatz sein, wenn es darum geht, das Potenzial von technischen Neuerungen oder die Wirkung von kurzfristig orientierten und lokal begrenzten Eingriffen auszuloten. Worin genau liegt der Charme dieses Ansatzes? Um die Frage nach der ursächlichen Wirkung eines Eingriffs zu beantworten, können Natur- und Technikwissenschaftler/innen oft die kontrollierte Studienumgebung eines Labors nutzen. Dies erlaubt ihnen, ein Studiendesign zu verfolgen, das den Eingriff von anderen Einflüssen isoliert. Die zu untersuchende Maßnahme wird dann in ihrer Intensität variiert - im einfachsten Falle gibt es zwei „Regime“: eines mit und eines ohne die Maßnahme. Die damit einhergehenden Unterschiede in den betrachteten Ergebnisgrößen werden als ursächliche Wirkung dieser Variation festgehalten.

Es geht also um etwas viel Anspruchsvolleres als um das für sich genommen sicherlich häufig ebenfalls interessante bloße „Ausprobieren“ einer Neuerung: Experimentelle Ansätze erlauben es im Grundsatz, kluge Fragen nach dem „Was wäre, wenn?“ zu stellen. Die kontrollierten Studienbedingungen eines Labors innerhalb der „im Feld“ herrschenden Lebenswirklichkeit in einem „Reallabor“ herzustellen, ist daher zumindest im Grundsatz attraktiv. Die hohe analytische Aussagekraft (interne Validität) des experimentellen Ansatzes ermöglicht Erkenntnisse mit Relevanz für politische Entscheidungsträger freilich nur dann, wenn die Bedingungen des Reallabors so gestaltet sind, dass sie sich auch überzeugend in die Lebenswirklichkeit außerhalb des Reallabors übertragen lassen (externe Validität).

\section{... und ihre Grenzen}

Wenn der Erkenntnisgewinn über das bloße Ausprobieren hinausgehen soll, sind Erfahrungen im Reallabor erstens dann analytisch wertlos, wenn es nicht gelingt, das Reallabor hinreichend von der Außenwelt zu isolieren. Über das Reallabor müsste mithin eine Art Käseglocke gestülpt werden, die verhindert, dass Akteure von außerhalb ebenfalls die spezifischen Regelungen innerhalb des Reallabors nutzen können. Gleichzeitig müsste das Leben innerhalb des Reallabors aber weitgehend so ablaufen, wie es auch im „Normalbetrieb“ der Lebenswirklichkeit der Fall ist. Dieser Balanceakt ist selbst im besten Falle äußerst herausfordernd. Zweitens erlauben die Erfahrun- gen im Reallabor keine tiefere Aussage zu allen Eingriffen oder Veränderungen, die in der Lebenswirklichkeit ortsübergreifend angelegt sind, etwa zur Einrichtung überregionaler Netzwerke.

Schließlich sind Reallabore nicht dazu geeignet, die Wirksamkeit von umfassenden, systemischen Regulierungselementen oder deren Reform zu beurteilen. Derartige Maßnahmen greifen in ein arbeitsteiliges, meist Regionen überspannendes wirtschaftliches Geschehen ein und lassen sich schon aufgrund dieser übergreifenden Natur nicht lokal erproben. Denn marktwirtschaftlich verfasste Volkswirtschaften zeichnen sich durch ein hohes Maß an Arbeitsteilung aus, die über Märkte moderiert wird und so die Handlungen von Akteuren verknüpft, die sich physisch nie begegnen müssen.

Als Faustregel gilt: Je umfassender und systemischer eine Veränderung ausfällt und je längeren Atem der ersehnte Wandel erfordert, umso schwerer ist es, den gesuchten Kontrast mit und ohne Eingriff in der engen räumlichen und zeitlichen Abgrenzung eines Reallabors zu konstruieren. Der tief greifende Wandel zu einer nachhaltigen Wirtschafts- und Lebensweise wird sich daher nicht im Reallabor vorwegnehmen und evaluieren lassen. Umso mehr kommt es darauf an, dass im Einklang mit der ersten Forderung der vorsorgeorientierten Postwachstumsposition die Rahmenbedingungen in der (internationalen) Realität möglichst schnell und umfassend „richtig“ gesetzt werden. Im Sinne der dritten Forderung sollten Politik und Gesellschaft zugleich danach streben, durch den effizienten Einsatz der materiellen Basis ein möglichst hohes Maß an individueller Lebensqualität und gesellschaftlichem Wohlergehen innerhalb der planetaren Belastungsgrenzen zu verwirklichen.

\section{Literatur \\ Appelrath, H./Büdenbender, U./Edenhofer, O./Haucap, J./Knopf, B./Lange, T./Mayer, C./Rehtanz, C./ Schmidt, C. M./Themann, M. (2015): Die Energiewende europäisch integrieren. Neue Gestaltungsmöglichkeiten für die gemeinsame Energie- und Klimapolitik. In: Schriftenreihe zur wissenschaftsbasierten Politikberatung, München, acatech. \\ Edenhofer, O./Schmidt, C. M. (2018): Eckpunkte einer $\mathrm{CO}_{2}$-Preisreform. Essen, RWI - Leibniz-Institut für Wirtschaftsforschung (Hrsg.), RWI Position Nr. 72. \\ Hielscher, K./Enkelmann, S. (2018): Reallabore in Deutschland - Forschungs- agenda zur Entwicklung eines Handbuchs für regulatorische Experi- mentierräume. In: BMWi (Hrsg.): Schlaglichter der Wirtschaftspolitik, Monatsbericht März 2018: 5-6. \\ Petschow, U./aus dem Moore, N./Pissarskoi, E./Korfhage, T./Lange, S./ Schoofs, A./Hofmann, D. (2018): Gesellschaftliches Wohlergehen inner- halb planetarer Grenzen: Der Ansatz einer vorsorgeorientierten Post- wachstumsposition. UBA Texte 89/2018.}

\section{AUTOR + KONTAKT}

Dr. Christoph M.Schmidt ist Präsident des RWI Leibniz-Institut für Wirtschaftsforschung, Vorsitzender des Sachverständigenrates zur Begutachtung der gesamtwirtschaftlichen Entwicklung (SVR) sowie Professor an der Ruhr-Universität Bochum.

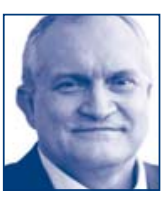

RWI, Hohenzollernstr. 1-3, 45128 Essen. Tel.: +49 201 8149227, E-Mail: praesident@rwi-essen.de 